\title{
THE USAGE OF MEDICAL INFORMATICS IN CRITICAL CARE MEDICINE
}

\section{Type of article: conference abstract}

\author{
Marsa Gholamzadeh1*, Hamidreza Abtahi2 \\ 1: Master Student in Medical Informatics, School of Allied Medicine, TehranUniversity of \\ Medical Sciences and Thoracic Research Center, Tehran, Iran. \\ 2: Associate professor, Department of pulmonary and critical care medicine, Thoracic \\ Research Center, Tehran University of Medical Sciences, Iran. \\ * Tel: +989122450672, E-mail: m-gholamzadeh@razi.tums.ac.ir
} \begin{abstract}
Introduction: The intensive care unit can be defined as a complex system that composed of clinical informations, tasks and knowledge. It is also one of the most stressful and most vital parts of healthcare centers which involve a huge amount of information and clinical data daily. These informations should be analyzed and managed in the best way as soon as possible to restore patients to normal by health professionals. Accurate and timely diagnosis, the best treatment and to avoid any possible error in this section can be equivalent to achieve the best possible result and reducing the length of hospitalization and mortality. As a result, due to the complex nature of the critical care and the mass of clinical data; using a combination of medical knowledge with the latest technologies and use of medical informatics capabilities can be the best way to reduce the workload of the sector and improve the quality of patient care.
\end{abstract}

Methods: A systematic search was conducted on the PubMed/MEDLINE, web of science, BMJ, ScienceDirect, and Scopus database for finding studies that have related to critical care and usage of informatics or medical informatics. The collected data and results are summarized by researchers and the results analyzed based on similarities and differences.

Results: With increased development of medical informatics and electronic systems has led to substantial progress in the field of critical care since 1980 till now around the world. Several articles, research projects has published. From 1981 to 2016 almost 600 scientific papers were found which included reports, posters and thesis written in context of critical care medical informatics. More than 65 percent of these studies is about designing CDSS in this field. Today, many of these systems are used in medical centers around the world and lead to improving the quality of patient care and reduce medical errors in intensive care units. Among these 600 papers, 230 articles related to our issue in fields of the design of electronic records, clinical information systems, decision support systems, data mining, telemedicine, smart analysis of clinical information and knowledge extraction techniques were selected as the main source of this study.

Conclusion: According to results of our study, it seems that critical care is potentially a valuable resource for medical informatics researches. The applied of medical informatics in the different fields of the diagnosis, interpretation, and treatment in different countries have improved the quality of care for patients in critical care field. We can mention some common fields which used such as infection control and early detection in intensive care units, clinical information systems, and CPOE and decision support systems such as APACHE system for grading the severity of illness of patients who hospitalized and even telemedicine. Since this area of research has not been a field of interest in Iran yet, it seems that this study with the aim of review the application of medical informatics in different countries could lead to practical researches in this field.

KEYWORDS: Critical Care, Medical informatics, Critical care information systems

\section{Declaration of conflicts}

This abstract is selected from the First International Congress of Diseases and Health Outcomes Registry and First National Congress of Medical Informatics, 14-17 February 2017, Mashhad, Iran

\section{Authors' biography}

No biography. 
Medical Technologies Journal, Volume: 1, Issue: 4, October-December 2017, Pages:76-136. Doi : https://doi.org/10.26415/2572-004X-vol1iss4

\section{References}

No references. 